\title{
The impact of electromagnetic radiation on the degradation of magnetic ferrofluids
}

\author{
Marek PaVlík ${ }^{1}$, Lukáš Kruželák ${ }^{1}$, Miroslav Mikita ${ }^{1}$, Michal ŠPes ${ }^{1}$, \\ SAMuel Bucko ${ }^{1}$, LukÁŠ Lisoñ ${ }^{1}$, Michal Kosterec ${ }^{1}$, L'Ubomír BeŇA ${ }^{1}$, Pavol Liptai ${ }^{2}$ \\ ${ }^{1}$ TUKE FEI, Department of Electric Power Engineering \\ Mäsiarska 74, 04200 Košice, Slovakia \\ ${ }^{2}$ TUKE SjF, Department of process and environmental engineering, \\ Letna 9, 04200 Kosice, Slovakia \\ e-mail: \{marek.pavlik, lukas.kruzelak, miroslav.mikita, michal.spes, samuel.bucko, lukas.lison, \\ michal.kosterec,lubomir.bena, pavol.liptai\}@tuke.sk
}

(Received: 02.08.2016, revised: 28.01.2017)

\begin{abstract}
This article deals with magnetic nano-fluids, which are the part of transformer oil ITO 100 and their behavior is influenced by a permanent magnetic field. We performed an IRC analysis in the time domain on the three different samples. Measurements were made before and after radiation of an electromagnetic field. The main objective was to examine changes in the properties of the samples due to the influence of the electromagnetic field. The measurements depend on the orientation of the external magnetic field. This behavior occurs especially during the structuring of the nanoparticles in the sample exposed to the magnetic field. These processes change the polarization of the liquid because the nanoparticles concentration is contained in the fluid.
\end{abstract}

Key words: ferrofluids, electromagnetic radiation, oxidation stability, transformer oil

\section{Introduction}

The condition of the insulation is an essential aspect for the operational reliability of power transformers, generators, cables and other high voltage equipment. The power transformer is expensive and vital equipment in the transmission and distribution grid and its life is predominantly determined by the condition of the insulation system. During its operation, this system is continuously subjected to thermal, electrical and chemical stresses etc. [1,5].

Electrical insulation and the heat transfer are important properties of the liquids used in the transformers. New liquids, e.g. silicone or esters, are used in the special-purpose transformers. These liquids such as seed-oil-based ones are more environmentally friendly, but absorb more moisture. Actually, a large majority of the transformers have their insulation systems consisting of the cellulose paper and the mineral oil. The mineral oil has good oxidation stability and a poor moisture absorbing property due to inhibitors, but his disadvantage rests in the low 
thermal conductivity and this factor has an influence on the thermal loading property of the device $[2,4]$. From the chemical point of view, the mineral oil is a mixture of the naphthenic, paraffinic and aromatic hydrocarbons. A fire point of mineral oil is approximately $160^{\circ} \mathrm{C}$, which is lower than the fire point of ester - approximately $300^{\circ} \mathrm{C}$ [3]. It was provided by comparison of the mineral oil with the vegetable oil. When compared to vegetable oils, permittivity measured for the new mineral oil was $\varepsilon_{r}=2.9$. New environmentally friendly products and limited petroleum resources are an impulse for many researchers to replace mineral oils by vegetable oils or to improve the thermal and insulating properties of mineral oils. For the improvement of the cooling and isolation properties, the transformer based magnetic nano-fluid (MN) was proposed and tested. Ferrofluid [8] is one of such MNs.

The ferrofluids exhibit special properties that make them suitable for many technical and medical applications. Some of these properties are determined by the particular physics of small-sized magnetic nanoparticles [18]. Therefore, it is important to search for better methods and theories describing the physical behavior of these liquids, and in particular, induced structuring of magnetic particles $[12,15]$.

Many physical properties of the magnetic fluids observed while influenced by the magnetic field reveal, that clusters of constituent magnetic particles exist, which results in various anisotropic properties [16]. Therefore, an understanding of the clustering phenomena in magnetic fluids is important when explaining the physical properties of magnetic fluids while being subjected to the external electromagnetic field $[11,14]$.

These ferrofluids consist of the carrier fluid (transformer oil), nanoparticles and a hydrophobic single layer of oleic acid as a surfactant. The surfactant has a role of covering the nanoparticles and it prevents their contact from each other, thus the creation of clusters. Magnetite $\left(\mathrm{Fe}_{3} \mathrm{O}_{4}\right)$ was used as nanoparticles. The creation procedure was developed in the article [7]. To estimate the effective electric permittivity of $M N \varepsilon_{M F}$ the Maxwell-Garnett formula was used for a mixture with spherical inclusions [8].

$$
\varepsilon_{M F}=\varepsilon_{I T O}+3 \varphi \varepsilon_{I T O} \frac{\varepsilon_{N P}-\varepsilon_{I T O}}{\varepsilon_{N P}+2 \varepsilon_{I T O}-\varphi\left(\varepsilon_{N P}-\varepsilon_{I T O}\right)} .
$$

where: $\varepsilon_{I T O}$ and $\varepsilon_{N P}$ are the electric permittivity of the transformer oil and nanoparticles of magnetite. The $\varphi$ is a solid volume fraction of magnetite nanoparticles [17].

In the article [9], EMR is exactly used for thorough dispersion of nanoparticles while producing ferrofluid with a carrier fluid (ester). After the thermal aging of the samples, it is proved that the surfactant degrades and the particles start to agglutinate into the clusters $[10,15]$. In the case of insulating liquids this phenomenon is undesirable because of polarizability and the resultant breakdown of dielectric material that has a destructive impact on the transformer $[13,19]$.

This fact is a motivation for this article, where the impact of EMC radiation on the ferrofluid and potential partial breakdown of the nanopartical clusters are observed. This phenomenon may partially revitalise or accelerate degradation of the ferrofluid. The revitalization is observed by the time domain spectroscopy (polarization and depolarization current) $[10$, 16-17]. 


\section{General knowledge about the experimental method}

With time domain spectroscopy, it is possible to investigate the slow polarization processes. It is a way of measuring the polarization and depolarization of currents (PDC) with a DC voltage step [6].

The electric field is generated by an external voltage $u(t)$ and it is applied on the dielectric material. The current through the dielectric material can be expressed as (2)

$$
i(t)=C_{0}\left[\frac{\sigma}{\varepsilon_{0}} u(t)+\varepsilon_{r} \frac{\mathrm{d} u(t)}{\mathrm{d} t}+\frac{\mathrm{d}}{\mathrm{d} t} \int_{0}^{t} f(t-\tau) u(\tau) \mathrm{d} \tau\right],
$$

where, $C_{0}$ is the geometrical capacitance of the dielectric material, $u(t)$ is the step voltage, $\sigma$ is the DC conductivity of the dielectric material, $\tau$ is the time constant, $\varepsilon_{0}=8.852 \cdot 10^{-12} \mathrm{~F} / \mathrm{m}$ is the permittivity of a vacuum, $\varepsilon_{r}$ is the relative permittivity of the dielectric material and $f(t)$ is the response function of the dielectric material [9].

As a result of the influence of the DC electrical field on the insulation, is the electric current influenced with conductive processes. This effect is characterized by leakage of the current and polarization processes, which are characterized by absorption of the current that flows through dielectric. Charging current $i_{n}(t)$ flowing through the dielectric is expressed by the formula:

$$
i_{n}(t)=i_{c}(t)+i_{a}(t)+i_{v},
$$

where: $i_{c}(t)$ is the capacitive component of the current, $i_{a}(t)$ is the absorption component of the current, $i_{v}$ iis the conductive component of the current.

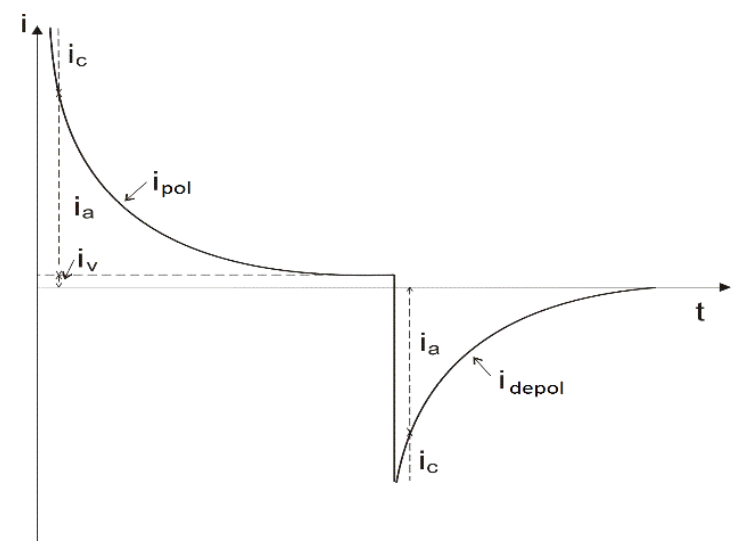

Fig. 1. Time course of the charging and discharging currents [4]

The total current $i(t)$ flowing through the dielectric can be expressed as the sum of all elementary relaxation currents. It exponentially decreases over time and is determined as: 


$$
i(t)=\frac{U}{R}+\sum_{i=1}^{n} I_{m i} \exp \left(\frac{-t}{\tau_{i}}\right)
$$

where: $U$ is the applied direct voltage, $R$ is the insulation resistance over $\infty$ time, $I_{m i}$ is the amplitude of the $i$-th elementary current, $\tau_{i}$ is the time constant of the $i$-th elementary current, $n$ is the number of elementary currents [11]

\section{Realization of experiment}

The electric power transformers are sometimes placed in the vicinity of transmitters for mobile communications and their radiation can affect these transformers. By using the conventional transformer oil the electromagnetic radiation has no effect. Power engineering develops new and better insulation materials, such as magnetic fluid, that is one of them. In the future, magnetic fluids could replace the transformer oil. Our aim was to determine whether high frequency of electromagnetic field radiation had any influence on the magnetic fluids. Therefore, we have chosen a frequency of $900 \mathrm{MHz}$ since it is used by mobile operators.

The aim of the measurement was to determine the impact of the electromagnetic field on magnetic ferrofluids at room temperature $\left(20^{\circ} \mathrm{C}\right)$ without the influence of an external electromagnetic field. For the measurement, the KEITHLEY K617 device was used with a measuring voltage of $100 \mathrm{~V}$ intended for measurements of charging and discharging currents. The measuring devices and the circuit configuration is shown in Fig. 2.

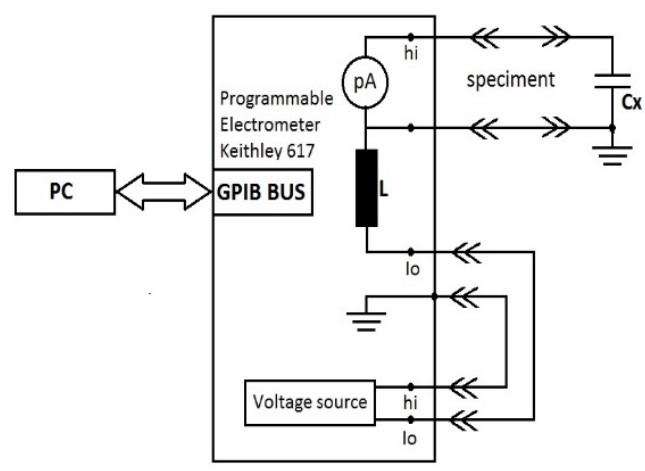

Fig. 2. Circuit configuration for measurement

Used measuring devices are very susceptible to electromagnetic interference (EMI). For that reason, measurements were carried out in the non-reflective EMC chamber, which prevents against permeability of EMI from the environment. Three selected samples of magnetic fluids were submitted to PDC analysis, while the charging and discharging currents were measured. The measurements were automated by the Agilent VEE software. The measured values were recorded during the measurements. The magnetic fluid has been drawn up in concentrations that are expressed using magnetization. A manufacturer doesn't define the volu- 
metric representation of particles in magnetic fluid and defines only the size of magnetization of individual samples. The labeled samples were called Gauss 10, Gauss 25 and Gauss 50 and the concentration of particles in the individual magnetic fluids did not exceed $5 \%$. The container, which is shown in Fig. 3 and which was used for measurements, contains the system of flat electrodes.

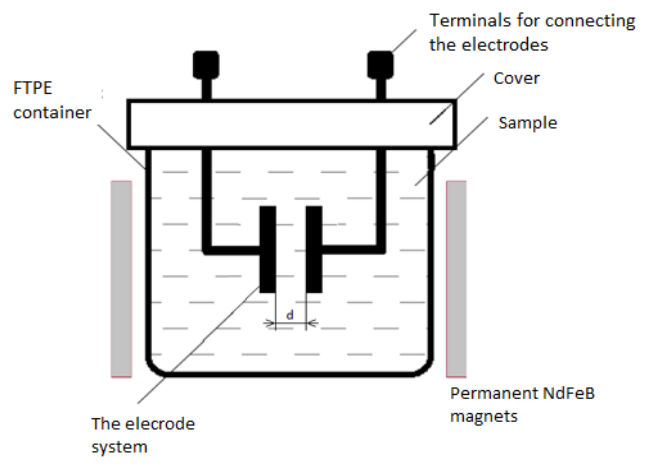

Fig. 3. Preparation for measurement of samples

The used container should have a volume of approximately $75 \mathrm{ml}$ with the two circular electrodes. The diameter of each electrode was $20 \mathrm{~mm}$ and the distance between the electrodes was $1 \mathrm{~mm}$.

Afterwards, we have measured individual samples which were exposed to the electromagnetic field. Each measurement was repeated for comparison of the results.

The samples were placed in a non-reflective EMC chamber because of exterior influences. The antenna was connected with Analog Signal Generator Agilent MXG N5183A. The power amplifier was connected to an analog signal generator. Radiation power of electromagnetic field was set at $30 \mathrm{~W}$ at a frequency of $900 \mathrm{MHz}$. All samples were irradiated during 100 hours.

The measurements of the individual samples were repeated three times. The resulting values of charging and discharging currents were averaged.

\section{Evaluation of measured results}

At processed results, there can be seen that the curve of charging currents is slightly deformed. The size of the bending depends on the percentage of magnetic particles in the magnetic fluid. These anomalies are probably caused by dielectrophoresis.

For the sample GAUSS 10, the anomalies are much smaller than for the other samples. This is due to the fact, that concentration of magnetic particles is lower in this particular sample and therefore it creates less chains which has a great influence on the charging current of the samples.

When comparing the charging currents of irradiated and non-irradiated samples, it is clear that the irradiated sample shows more anomalies than the non-irradiated sample. Increased 
occurrence of these anomalies is likely to be caused by irradiation of the samples. This radiation has an influence on the magnetic particles contained in the magnetic fluid.

\section{GAUSS 10 charging current}

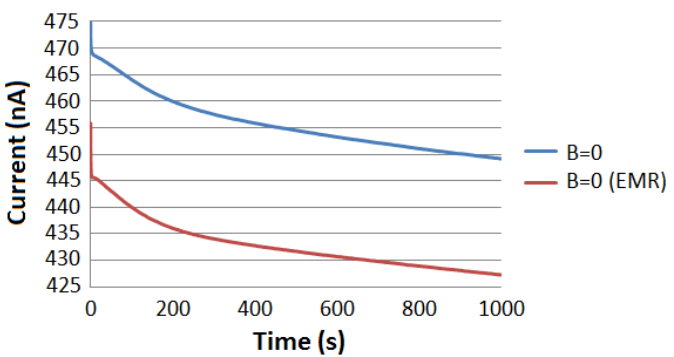

Fig. 4. The graphic dependence of the charging current on the time, the sample GAUSS 10 before the influence of electromagnetic radiation (EMR) and after the influence of EMR

This electromagnetic radiation has a high frequency and power. The magnetic particles under the influence of an electromagnetic field are heated and these overheated magnetic particles release the chains of surfactant. These surfactants deteriorating the properties of the magnetic fluid.

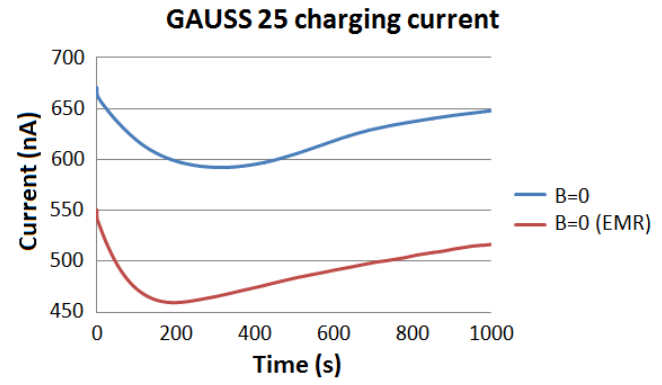

Fig. 5. The graphic dependence of the charging current on the time, the sample GAUSS 25 before the influence of EMR and after the influence of EMR

The volumetric representation of magnetic particles is much greater in the samples GAUSS 50 and GAUSS 25 than in the sample GAUSS 10 and the volume of surfactants is greater, too.

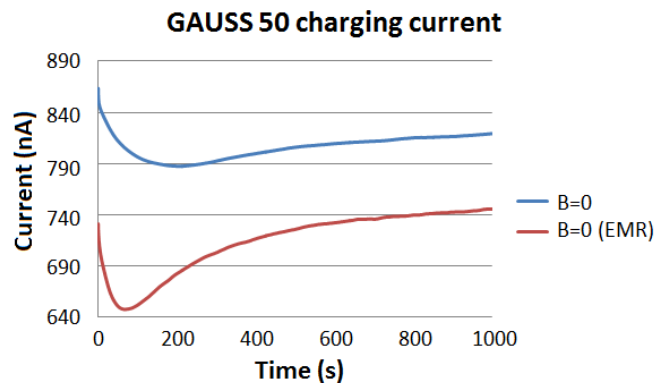

Fig. 6. The graphic dependence of the charging current on the time, the sample GAUSS 50 before the influence of EMR and after the influence of EMR 
From graphic results of discharging currents in the individual samples, it can be seen that the discharging in the sample GAUSS 10 is much steeper than in the samples GAUSS 25 and GAUSS 50. These differences are caused by chains that were formed while charging of the samples. These chains create a stable structure, causing that the depolarization of the samples was carried out slowly. This phenomenon is more noticeable in the samples GAUSS 25 and GAUSS 50, which is associated with the greater volumetric representation of the magnetic particles.

GAUSS 10 discharging current

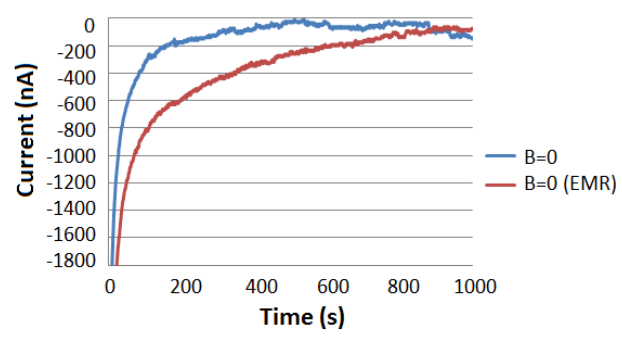

Fig. 7. The graphic dependence of the discharging current on the time, the sample GAUSS 10 before the influence of EMR and after the influence of EMR

When comparing the same samples before irradiation and after irradiation of the electromagnetic field, we can see the change in discharge characteristics of the current. The most significant change is observed in the sample GAUSS 10, this radiation has an impact on the magnetic particles, as well.

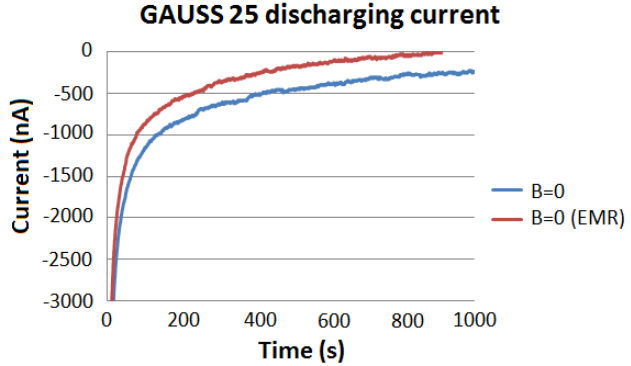

GAUSS 50 discharging current

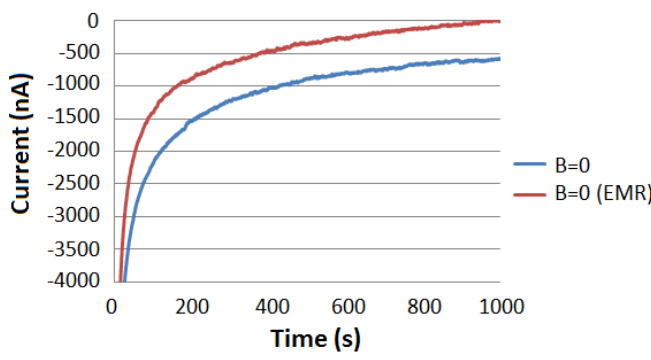

Fig. 8. The graphic dependence of the discharging current on the time, the sample GAUSS 25 before the influence of EMR and after the influence of EMR

Fig. 9. The graphic dependence of the discharging current on the time, the sample GAUSS 50 before the influence of EMR and after the influence of EMR 
As mentioned, under the influence of an electromagnetic field, the magnetic particles are heated up, that causes that weaker bond between the surfactant and the magnetic particles was disrupted. Increasing release of the amount of surfactant molecules in the magnetic fluid, caused deterioration of dielectric properties of the magnetic fluids.

The minor differences are observed in the irradiated and non-irradiated sample for samples GAUSS 25 and GAUSS 50, which are associated with the clusters formation of magnetic particles.

\section{Conclusion}

The main objective of this paper was to investigate whether electromagnetic radiation has an influence on the degradation of parameters of the magnetic fluids. In this experiment, three samples of magnetic fluids were used. To determine the state of liquid, PDC analyses were executed in the time domain. Individual samples were measured before and after the influence of an electromagnetic field. The results were graphically processed and compared. Experiments showed that electromagnetic radiation at a frequency of $900 \mathrm{MHz}$ and power of $30 \mathrm{~W}$ had a negative influence on the magnetic fluids. Since power transformers generate electromagnetic fields with a frequency of $50 \mathrm{~Hz}$, it would be advisable to verify experimentally the influence of the electromagnetic radiation at a frequency of $50 \mathrm{~Hz}$.

\section{Acknowledgements}

This work was supported by grant award FEI no. FEI-2015-6, The impact of electromagnetic fields on the properties of materials.

\section{References}

[1] Wolny S., Adamowicz A., Lepich M., Influence of temperature and moisture level in paper oil insulation on the parameters of the Cole-Cole model, IEEE Transactions on Power delivery, vol. 29, no. 1, pp. 246-250 (2014).

[2] Cimbala R., Kurimský J., Kolcunová I., Determination of thermal ageing influence on rotating machine insulation system using dielectric spectroscopy, Przegląd Elektrotechniczny, vol. 87, no. 8, pp. 176-179 (2011).

[3] Dolník B., Electromagnetic compatibility, TU of Košice (in Slovak), monography, pp. 36-64 (2013).

[4] Timko M., Marton K., Király J., Cimbala R. et al., Magneto-dielectric properties of transformer oil based magnetic fluids in the frequency range up to $2 \mathrm{MHz}$, Magnetohydrodynamics, vol. 48, no. 2, pp. 427-434 (2012).

[5] Zbojovský J., Mészáros A., Medved' D., Simulation of electromagnetic fields propagation through the various materials, Proceedings of 15th International Scientific Conference on Electric Power Engineering (EPE), Brno, Czech Republic, pp. 257-260 (2014).

[6] Bica D., Preparation of magnetic fluids for various applications, Romanian Reports in Physics, vol. 47, pp. 265-272 (1995).

[7] Medved’ D., Kanálik M., Addressing arrangement of the electromagnetic field in the vicinity of electric power lines, Elektroenergetika (in Slovak), vol. 8, no. 2, pp: 9-12 (2015). 
[8] Liptai P., Lukáčová K., The electromagnetic fields near a microwave oven - measurement and analysis, Annals of Faculty Engineering Hunedoara - International Journal of Engineering, vol. 14, no. 1, pp. 83-86 (2016).

[9] Du Y., Mamishev A.V., Lesieutre B.C., Zahn M., Kang S.H., Moisture solubility for differently conditioned transformer oils, IEEE Transactions on Dielectrics and Electrical Insulation, vol. 8, no. 5, pp. 805-811 (2001).

[10] Petráś J., Balogh J., Džmura J., Overvoltage protection in high-frequency technology, Proceedings of 13th International Scientific Conference on Electric Power Engineering (EPE) (in Czech), Brno, Czech Republic, pp. 893-897 (2012).

[11] Zbojovský J. et al., Effect of shielding materials for electromagnetic field distribution, Proceedings of 7 th International Scientific Symposium on Electrical Power Engineering (in Slovak), Stará Lesná, Slovak Republic, pp: 439-442 (2013).

[12] Cimbala R., Kolcunová I., Dedinska L., Cackova V., Thermal dependency of dielectric properties of liquid insulating materials, Proceedings of 11th International Scientific Conference on Electric Power Engineering (EPE), Brno, Czech Republic, pp. 743-747 (2010).

[13] Kanálik M., Kolcun M., Computation of harmonic flows in three-phase systems, Acta Electrotechnica et Informatica, vol. 7, no. 3, pp. 41-45 (2007).

[14] Liptai P., Measurement methodology and evaluation of high-frequency electromagnetic fields of base stations of mobile operators in populated areas Ukraine - EU, Modern Technology, Business and Law (in Slovak), pp. 306-309, (2016).

[15] Pavlík M., Kolcunová I., Dolník B., Kurimský J., Mészáros A., Medved’ D., Kolcun M., Zbojovský J., Measuring of dependence of shielding effectiveness of wet materials on the frequency of electromagnetic field in the high frequency range, Acta Electrotechnica et Informatica, vol. 13, no. 3, pp. 12-16 (2013).

[16] Medved' D., Hirka O., Impact of electromagnetic fields in residential areas, Elektroenergetika, vol. 9, no. 2, pp. 13-16 (2016).

[17] Zbojovský J., Mészáros A., Kurimský P., Modelling the high frequency electromagnetic field propagation through the polystyrene, Proceedings of 8th International Scientific Symposium on Electrical Power Engineering, Stará Lesná, Slovak Republic, pp. 556-559 (2015).

[18] Marton K., Tomčo L., Cimbala R. et al., Magnetic fluid in ionizing electric field, Journal of Electrostatics, vol. 71, no. 3, pp. 467-470 (2013).

[19] Rajnak M., Timko M., Kopcansky P., Paulovicova K et al., Structure and viscosity of a transformer oil-based ferrofluid under an external electric field, Journal of Magnetism and Magnetic Materials 2016, doi: 10.1016/j.jmmm.2016.10.008 